\title{
Transforming ATTR cardiac amyloidosis into a chronic disease: The enormous potential of quantitative SPECT to improve diagnosis, prognosis, and monitoring of disease progression
}

\author{
Michael P. Ayers, MD, ${ }^{\mathrm{a}}$ Adithya V. Peruri, $M D,{ }^{\mathrm{a}}$ and \\ Jamieson $M$. Bourque, $M D, M H S^{a, b, c}$ \\ a Division of Cardiovascular Medicine and the Cardiac Imaging Center, University of Virginia \\ Health System, Charlottesville, VA \\ b Department of Radiology and Medical Imaging, University of Virginia Health System, \\ Charlottesville, VA \\ c Medical Director of Nuclear Cardiology and the Stress Laboratory, Division of Cardiovascular \\ Medicine and the Cardiac Imaging Center, University of Virginia Health System, Charlottesville, \\ VA
}

Received Feb 1, 2021; accepted Feb 1, 2021

doi: $10.1007 / \mathrm{s} 12350-021-02587-w$

See related article, pp. 1835-1845

A handful of slides.... that was the extent of my medical school education on cardiac amyloidosis. An accumulation of misfolded precursor proteins deposited in extracellular tissue, amyloid comprised a group of systemic illnesses with myriad presentations. ${ }^{1}$ In the past, diagnosis of cardiac amyloidosis required a biopsy, or often multiple biopsies, and was usually recognized late in the course of illness. Once diagnosed, the disease itself proved difficult to manage, with significant morbidity and mortality. Worse still, there were no disease modifying therapies, with only supportive care to offer. ${ }^{2}$ Fast-forward a decade plus, and the tables are turning. Disease modifying agents target the very biochemistry of the pathogenic proteins. ${ }^{3,4}$ Our newfound ability to

\footnotetext{
Funding No funding source.

Reprint requests: Jamieson M. Bourque, MD, MHS, Medical Director of Nuclear Cardiology and the Stress Laboratory, Division of Cardiovascular Medicine and the Cardiac Imaging Center, University of Virginia Health System, 1215 Lee Street, PO Box 800158Charlottesville, VA 22908; jamieson2@gmail.com

J Nucl Cardiol 2021;28:1846-50.

$1071-3581 / \$ 34.00$
}

Copyright (c) 2021 American Society of Nuclear Cardiology. alter the course of this highly morbid disease and change a patient's course of illness provided a moral imperative to improve the rate of diagnosis, strive for earlier recognition of disease, and identify mechanisms to surveil treatment progress. To fill this void, radionuclide imaging has stepped in, revolutionizing accurate noninvasive approaches for a patient population in need. As obtaining a diagnosis became less invasive, and treatments offered genuine promise, it became increasingly apparent that amyloidosis may not be as rare as once thought. ${ }^{5}$ Some recent studies have estimated that upwards of $10 \%$ of patients with heart failure with preserved ejection fraction may have a form of amyloidosis. $^{6}$

Clinical clues that may lead to a diagnostic workup for ATTR include increasing age, a history of early onset carpal tunnel syndrome in a patient without an occupational risk factor, early onset of spinal stenosis due to thickening of the ligamentum flavum, history of distal bicep tendon rupture, premature hip or knee arthroplasty, heart failure with preserved ejection fraction in a patient without any history of hypertension, low-flow low-gradient severe aortic stenosis, or a latein-life diagnosis of hypertrophic cardiomyopathy. ${ }^{1,7-9}$ If clinical features are present that suggest amyloidosis as a possibility, electrocardiography and imaging with echocardiography, and when needed, cardiac magnetic resonance imaging (CMR) are useful imaging tools to 
increase suspicion or rule-in alternative diagnoses. Yet, while they can suggest the disease, none of these modalities can confirm a diagnosis of ATTR cardiac amyloidosis.

Historically, after a suggestive clinical presentation or suspicious findings on echocardiography, or CMR, the next step in the workup for amyloidosis would have been biopsy with Cong Red staining. A noninvasive method of disease confirmation, however, is now available. Radionuclide imaging with $99 \mathrm{~m}$-Tc-labeled bisphosphonate or pyrophosphate (PYP) compounds has remarkably high sensitivity for ATTR cardiac amyloidosis. Current tracers available include ${ }^{99 \mathrm{~m}}$ Tc-PYP, ${ }^{99 \mathrm{~m}}$ Tc-DPD, and ${ }^{99 \mathrm{~m}}$ Tc-HMDP. ${ }^{5}$ The predominant tracer used in the U.S. is ${ }^{99 m}$ Tc-PYP. Absent of pathology, these bone tracers should not demonstrate myocardial uptake. In the presence of amyloid fibrils and associated microcalcifications, myocardial uptake will occur. The latest ASNC-led multi-societal expert consensus recommendations for multimodality imaging in cardiac amyloidosis (see Fig. 1), state that radionuclide imaging is now strongly preferred as the next step after a suggestive echocardiogram or CMR study. ${ }^{10,11}$

ATTR cardiac amyloidosis, for unknown reasons, has a greater propensity to cause microcalcifications than does AL. ${ }^{5}$ Due to the lower levels of microcalcifications with AL, ${ }^{99 \mathrm{~m}}$ Tc-PYP/DPD/HMDP radionuclide imaging has only moderate sensitivity to detect AL disease. Therefore, in the setting of a mild or moderately positive ${ }^{99 m}$ Tc-PYP/DPD/HMDP study, one cannot clearly distinguish AL from ATTR cardiac amyloidosis. For this reason, a positive ${ }^{99 \mathrm{~m}} \mathrm{Tc}-\mathrm{PYP} / \mathrm{DPD} / \mathrm{HMDP}$ radionuclide test requires further workup. All patients undergoing testing for ATTR cardiac amyloidosis must also have AL amyloidosis ruled-out. This can be performed by assessing for presence of a monoclonal protein by quantitating serum free light chains and performing serum and urine electrophoresis with immunofixation. ${ }^{12}$ This two-step approach of ${ }^{99 \mathrm{~m}} \mathrm{Tc}$ PYP/DPD/HMDP radionuclide imaging and laboratory evaluation for AL amyloidosis, summarized in the ASNC-led consensus (Fig. 1), has significantly reduced the need for invasive endomyocardial biopsy. ${ }^{10}$

The endorsed method of interpretation of ${ }^{99 \mathrm{~m}} \mathrm{Tc}$ PYP/DPD/HMDP bone scintigraphy studies in the latest expert consensus recommendations begins with confirmation of diffuse radiotracer uptake in the myocardium on single-photon emission computed tomography (SPECT) and planar images. If uptake is present, semiquantitative visual grading is performed in which

Heart failure, syncope, or bradyarrhythmia, with echocardiogram and/or cardiac magnetic resonance imaging (CMR) suggesting/indicating cardiac amyloid

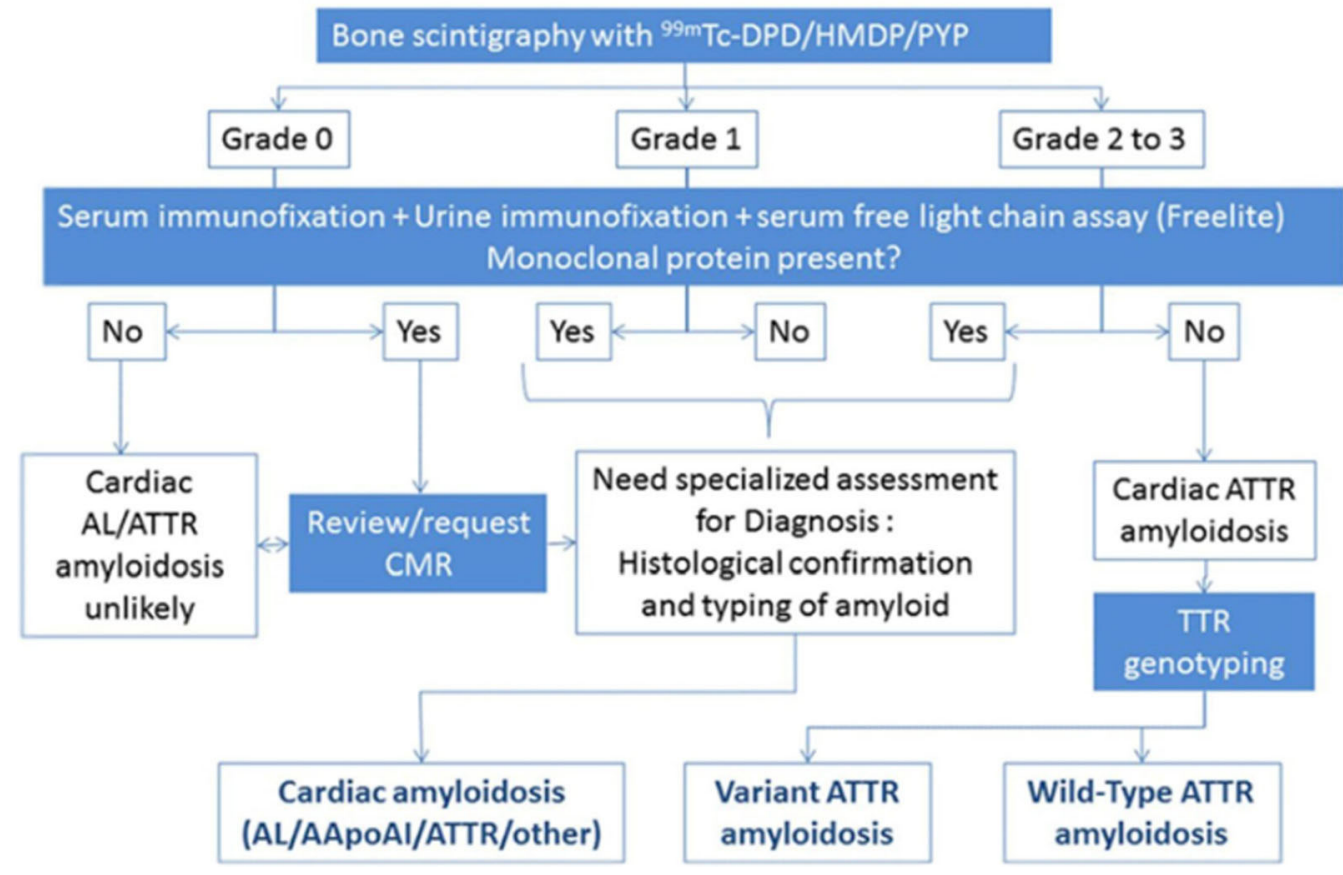

Fig. 1. Diagnostic algorithm for cardiac amyloidosis. 
myocardial uptake is compared to the surrounding ribs. A score of 0 refers to no tracer uptake, whereas a score of 3 refers to uptake greater in the myocardium than the surrounding ribs. Grade 2 or 3 uptake confirms ATTR cardiac amyloidosis (with negative AL lab testing) and grade 0 excludes the diagnosis. Grade 1 uptake is borderline and can be present in other forms of amyloidosis, may be present in early disease, or may even be normal.

Compared to 2-dimensional planar scintigraphy, SPECT allows for improved localization of radiotracer uptake. Gamma cameras acquire planar images from multiple angles and reconstruct three dimensions. By integrating computed tomography, SPECT also allows for attenuation correction, further improving diagnostic accuracy. Particularly in states of low cardiac output, distinguishing residual radiotracer in the blood pool from uptake in the myocardium can prove challenging. By providing a three-dimensional image, SPECT decreases the likelihood of falsely classifying residual blood pool activity as myocardial uptake, decreasing false positive tests and further improving positive predictive value. In direct comparisons of planar and SPECT imaging for amyloidosis, SPECT not only decreases rates of equivocal quantitation, it results in reclassification of $16 \%$ of planar scans. ${ }^{13}$ There is already considerable utilization of SPECT imaging for ischemia. By increasing the reliability of imaging interpretation in an already familiar imaging modality, SPECT presumably would increase the ability of lower volume nuclear laboratories to reliably and accurately diagnose ATTR. ${ }^{14}$

As with most subjective scales, the interobserver reliability proves most challenging in differentiating intermediate scores. Qualitative scores may demonstrate improved inter-observer variability in differentiating a visual grade of 1 from a visual grade of 2. For this reason, semi-quantitative measurements are recommended to improve diagnostic accuracy. ${ }^{10} \mathrm{~A}$ heart to contralateral lung (H:CL) ratio calculates the mean counts in a region of interest (ROI) around the heart relative to a ROI in the contralateral lung. If performed and interpreted correctly, the semi-quantitative H:CL ratio of $\geq 1.5$ accurately identifies ATTR cardiac amyloidosis. ${ }^{12}$ While semi-quantitative scoring has largely proven reproducible from center to center with standardization of scan acquisition timing at 1 and 3 hour intervals, it is not without challenges. ${ }^{10}$ The most common pitfall is calculating a heart ROI in the setting of intense blood pool uptake rather than myocardial uptake. A hyperintense blood pool can falsely elevate a H:CL ratio. Conversely, it is possible to incorrectly creating an area of interest for comparison to the heart. If an ROI is constructed in the contralateral lung that includes a rib fracture, other area of avid bone uptake, or the right ventricle, the corresponding $\mathrm{H}: \mathrm{CL}$ ratio would be artificially low, resulting in a false negative. ${ }^{15}$ Heart to whole body (H:WB) radios appears to have superior performance by receiver-operating curve analysis relative to $\mathrm{H}: \mathrm{CL}$ ratios, with lower rates of false negative tests, ${ }^{16}$ but are less frequently employed.

Reproducible diagnostic accuracy with qualitative and semi-quantitative diagnosis of ATTR cardiac amyloidosis is an enormous step forward in the field. However, precise quantitation of amyloid burden is still an area of great unmet need. Semi-quantitative scoring severity does not clearly correlate with patient symptoms, risk stratification, or disease progression. ${ }^{5,15} \mathrm{Semi-}$ quantitative methods do not assess for extracardiac uptake, which may change myocardial ratios and alter diagnostic accuracy. ${ }^{17}$ Moreover, repeated radionuclide imaging with semi-quantitative assessment to monitor a patient's response to therapy has not been successful in an early study and is not common practice. ${ }^{15}$ In theory, fully quantitative methods should help to monitor disease progression and guide therapeutic decision making. A quantitative method could also allow study linking the extent of myocardial involvement and clinical course, supporting better prognostication and earlier diagnosis.

Assessment of cardiac amyloidosis with positron emission tomography (PET) has thus far shown the most promise in quantitative assessment, and there has been a high level of interest and academic investment in the utilization this imaging modality. While not in clinical use, at least 5 agents which directly bind to amyloid fibrils have been studied in PET: ${ }^{18} \mathrm{~F}$-flutemetamol, ${ }^{18} \mathrm{~F}$ florbetaben, ${ }^{11} \mathrm{C}$-PIB, ${ }^{18} \mathrm{~F}-\mathrm{NaF},{ }^{18} \mathrm{~F}$-florbetapir. ${ }^{18}$ These agents bind directly to amyloid, in distinct contrast to SPECT tracers which bind to associated microcalcifications rather than directly to fibrils. These tracers assess the whole body for amyloid involvement. Direct amyloid binding, combined with the proven superior spatial resolution of PET, provides a precise quantitation of total amyloid deposition burden. Some PET tracers assess amyloid as a whole and do not differentiate between AL and ATTR subtypes. However, ${ }^{18} \mathrm{~F}$-florbetaben has displayed promise differentiating AL from ATTR based on temporal changes in SUV. ${ }^{19}$ In small trial, ${ }^{11} \mathrm{C}$-PIB displayed $100 \%$ accuracy in differentiating AL from ATTR with semi-quantitative assessment, though use of the compound is limited by a 20-minute half-life. ${ }^{20}$ Use of this tracer could obviate the need for associated testing to rule out AL amyloidosis. Moreover, early studies demonstrate PET tracers may have the ability to prognosticate as well as diagnose. ${ }^{21-23}$ The primary limitations of PET are that it is less readily available in non-academic sites, requires expensive imaging hardware, and has more challenging tracer acquisition, often requiring on-site cyclotrons. Increased 
cost and radiation dose relative to SPECT are also reasonable concerns, especially as diagnostic workups for amyloidosis continue to increase worldwide. ${ }^{14}$

Given these challenges with use of PET as the primary imaging tool for cardiac amyloidosis assessment, there is strong interest in developing superior quantitative SPECT techniques. Relative to PET imaging, SPECT must overcome inherently inferior spatial resolution. Substantial attention has been paid to overcoming this limitation, including correction algorithms for attenuation and scattering, resolution recovery, and cross-calibration to produce results in a quantitative output in universal units $\left(\mathrm{kBq} \cdot \mathrm{ml}^{-1}\right) .{ }^{17}$ There have also been efforts to correct for instrumental dead time. ${ }^{17}$ Instrument dead time occurs when there is incomplete detection of photons from areas with extremely high photon emission, which varies from camera to camera and ultimately alters the relative distributions of radiotracer detected in the body. ${ }^{24}$ These quantitative units are also standardized for dose, injection time, and volume of distribution of radiotracer. Once standardized, they are used to create parametric maps of radiopharmaceutical distribution and intensity. ${ }^{17}$ These maps interlace with CT imaging for further improvement in localization and attenuation correction. ROIs are then drawn on structures such as the myocardium, blood pool, or bone that allow for direct comparison and quantitation of disease involvement.

Other established SPECT quantitation metrics include cardiac metabolic volume (CMV) and cardiac metabolic activity (CMA). ${ }^{17} \mathrm{CMV}$ is a straight-forward assessment of total myocardial involvement. CMA can be quantified using algorithms developed in oncology by identifying voxels above threshold values of activity. Recently, there has been increased interest in the use of Total Lesion Glycolysis (TLG), an oncology quantitation technique which multiplies cardiac metabolic volume by mean cardiac metabolic activity, giving weight to both volumetric burden and metabolic activity. These quantitation techniques still need to be further studied in larger trials to demonstrate reproducibility, prognostic value, and the ability to monitor disease progression, judge treatment response, and diagnose in earlier stages of disease. CMA and CMV have proven reproducible in high-uptake states, for example, but it is less clear how they will perform in mildly positive cases. $^{25,26}$

In this issue of the Journal, Miller et al. apply these SPECT quantitation metrics in a novel manner for application in ${ }^{99 m}$ Tc-PYP amyloid imaging. Their aim was to provide reproducible and accurate quantitation that provides similar value to PET. In that vein, Miller et al. examine the use of CPA and CMV (in their paper referred to as volume of interest, VOI) not only for diagnostic purposes, but also prognostically. Unsurprisingly, CPA and VOI demonstrate exceptional diagnostic capabilities, with receiver operating curves of 0.996 and 0.952, respectively. There was excellent interobserver agreement, which bodes well for more widespread reproducibility, including potentially at centers with less volume and expertise in SPECT imaging for ATTR cardiac amyloidosis. Perhaps more importantly, however, CPA was also associated with increased risk of reduced ejection fraction and hospitalization for heart failure. This correlation with meaningly clinical endpoints is an enormously important step in demonstrating the prognostic impact of SPECT imaging in ATTR cardiac amyloidosis.

This study was not without limitations. It was a single-center study with intermediate population size (124 patients), though this is not unexpected for this relatively rare disease. The trial was underpowered to detect cardiovascular death, a more meaningful endpoint. With respect to acquisition technique, algorithms to calculate CPA may vary between machines, decreasing reproducibility. The authors note that they utilized two different camera systems to acquire data, using varying acquisition times (12 versus 15 minutes). There are also inherent inaccuracies in SPECT quantitation of standard uptake values (SUV), due mostly to partial volume averaging. The authors cleverly addressed this limitation by using radiotracer counts in the blood pool rather than SUV when performing CPA calculations. $^{27}$

The use of non-invasive radionuclide imaging techniques for the evaluation of ATTR cardiac amyloidosis have been transformative for the field in a short period of time. With inevitable advancement, radionuclide cardiac imaging will move beyond simple diagnosis and early prognosis and will help guide cardiac amyloidosis treatment strategies. A reasonable future scenario could involve radionuclide assessment of the degree of amyloid deposition which in turn determines the appropriate medication class and therapy intensity. Radionuclide imaging is then utilized to monitor disease progression and response to therapy. With earlier diagnosis and tailored disease modifying therapies, ATTR cardiac amyloidosis can be converted from a lethal condition into a chronic disease. With improvements in prognosis, the correct patients can be shepherded towards advanced therapies sooner. Imaging, coupled with burgeoning treatment advances, has undeniably resulted in a surge in amyloid diagnoses and research. ${ }^{5}$ Cardiac amyloidosis has been transformed from a "rare disease,' traditionally covered in a matter of minutes during medical education curricula, into a promising area with great diagnostic and therapeutic potential. Advances in radionuclide imaging, and 
particularly quantitative techniques, will help lead the way forward.

\section{Disclosures}

Dr Bourque owns equity in Locus Health and is a consultant for Pfizer and General Electric.

\section{References}

1. Kittleson MM, Maurer MS, Ambardekar AV, Bullock-Palmer RP, Chang PP, Eisen HJ, et al. Cardiac amyloidosis: Evolving diagnosis and management: A scientific statement from the American Heart Association. Circulation 2020;142:e7-22.

2. Gertz MA, Dispenzieri A. Systemic amyloidosis recognition, prognosis, and therapy: A systematic review. JAMA 2020;324:7989.

3. Maurer MS, Schwartz JH, Gundapaneni B, Elliott PM, Merlini G, Waddington-Cruz $\mathrm{M}$, et al. Tafamidis treatment for patients with transthyretin amyloid cardiomyopathy. $\mathrm{N}$ Engl J Med 2018;379:1007-16.

4. Adams D, Gonzalez-Duarte A, O'Riordan WD, Yang CC, Ueda M, Kristen AV, et al. Patisiran, an RNAi therapeutic, for hereditary transthyretin amyloidosis. N Engl J Med 2018;379:11-21.

5. Ruberg FL, Grogan M, Hanna M, Kelly JW, Maurer MS. Transthyretin amyloid cardiomyopathy: JACC state-of-the-art review. J Am Coll Cardiol 2019;73:2872-91.

6. González-López E, Gallego-Delgado M, Guzzo-Merello G, de Haro-Del Moral FJ, Cobo-Marcos M, Robles C, et al. Wild-type transthyretin amyloidosis as a cause of heart failure with preserved ejection fraction. Eur Heart J 2015;36:2585-94.

7. Sekijima Y, Uchiyama S, Tojo K, Sano K, Shimizu Y, Imaeda T, et al. High prevalence of wild-type transthyretin deposition in patients with idiopathic carpal tunnel syndrome: A common cause of carpal tunnel syndrome in the elderly. Hum Pathol 2011;42:1785-91.

8. Rubin J, Alvarez J, Teruya S, Castano A, Lehman RA, Weidenbaum M, et al. Hip and knee arthroplasty are common among patients with transthyretin cardiac amyloidosis, occurring years before cardiac amyloid diagnosis: Can we identify affected patients earlier? Amyloid 2017;24:226-30.

9. Geller HI, Singh A, Alexander KM, Mirto TM, Falk RH. Association between ruptured distal biceps tendon and wild-type transthyretin cardiac amyloidosis. JAMA 2017;318:962-3.

10. Dorbala S, Ando Y, Bokhari S, Dispenzieri A, Falk RH, Ferrari VA, et al. ASNC/AHA/ASE/EANM/HFSA/ISA/SCMR/SNMMI expert consensus recommendations for multimodality imaging in cardiac amyloidosis: Part 2 of 2-Diagnostic criteria and appropriate utilization. J Nucl Cardiol 2020;27:659-73.

11. Gillmore JD, Maurer MS, Falk RH, Merlini G, Damy T, Dispenzieri A, et al. Nonbiopsy diagnosis of cardiac transthyretin amyloidosis. Circulation 2016;133:2404-12.

12. Dorbala S, Ando Y, Bokhari S, Dispenzieri A, Falk RH, Ferrari VA, et al. ASNC/AHA/ASE/EANM/HFSA/ISA/SCMR/SNMMI expert consensus recommendations for multimodality imaging in cardiac amyloidosis: Part 1 of 2-evidence base and standardized methods of imaging. J Nucl Cardiol 2019;26:2065-123.

13. Sperry BW, Burgett E, Bybee KA, McGhie AI, O'Keefe JH, Saeed IM, et al. Technetium pyrophosphate nuclear scintigraphy for cardiac amyloidosis: Imaging at 1 vs 3 hours and planar vs SPECT/CT. J Nucl Cardiol 2020;27:1802-7.

14. Singh V, Di Carli MF. SPECT versus PET myocardial perfusion imaging in patients with equivocal CT. Curr Cardiol Rep 2020;22:43

15. Castaño A, DeLuca A, Weinberg R, Pozniakoff T, Blaner WS, Pirmohamed A, et al. Serial scanning with technetium pyrophosphate $((99 \mathrm{~m}) \mathrm{Tc}-\mathrm{PYP})$ in advanced ATTR cardiac amyloidosis. J Nucl Cardiol 2016;23:1355-63.

16. Gallini C, Tutino F, Martone R, Ciaccio A, Costanzo EN, Taborchi $\mathrm{G}$, et al. Semi-quantitative indices of cardiac uptake in patients with suspected cardiac amyloidosis undergoing 99mTc-HMDP scintigraphy. J Nucl Cardiol 2019. https://doi.org/10.1007/s12350019-01643-w.

17. Ramsay SC, Cuscaden C. The current status of quantitative $\mathrm{SPECT} / \mathrm{CT}$ in the assessment of transthyretin cardiac amyloidosis. J Nucl Cardiol 2020;27:1464-8.

18. Kim YJ, Ha S, Kim YI. Cardiac amyloidosis imaging with amyloid positron emission tomography: A systematic review and metaanalysis. J Nucl Cardiol 2020;27:123-32.

19. Law WP, Wang WY, Moore PT, Mollee PN, Ng AC. Cardiac amyloid imaging with 18F-Florbetaben PET: A pilot study. J Nucl Med 2016;57:1733-9.

20. Lee SP, Lee ES, Choi H, Im HJ, Koh Y, Lee MH, et al. 11CPittsburgh B PET imaging in cardiac amyloidosis. JACC Cardiovasc Imaging 2015;8:50-9.

21. Minamimoto R, Awaya T, Iwama K, Hotta M, Nakajima K, Hirai $\mathrm{R}$, et al. Significance of (11)C-PIB PET/CT in cardiac amyloidosis compared with $(99 \mathrm{~m}) \mathrm{Tc}$-aprotinin scintigraphy: A pilot study. J Nucl Cardiol 2020;27:202-9.

22. Promislow SJ, Ruddy TD. The evolving landscape of nuclear imaging in cardiac amyloidosis. J Nucl Cardiol 2020;27:210-4.

23. Gallegos C, Miller EJ. Advances in PET-based cardiac amyloid radiotracers. Curr Cardiol Rep 2020;22:40.

24. Dorbala S, Ananthasubramaniam K, Armstrong IS, Chareonthaitawee P, DePuey EG, Einstein AJ, et al. Single photon emission computed tomography (SPECT) myocardial perfusion imaging guidelines: Instrumentation, acquisition, processing, and interpretation. J Nucl Cardiol 2018;25:1784-846.

25. Caobelli F, Braun M, Haaf P, Wild D, Zellweger MJ. Quantitative (99m)Tc-DPD SPECT/CT in patients with suspected ATTR cardiac amyloidosis: Feasibility and correlation with visual scores. J Nucl Cardiol 2020;27:1456-63.

26. Ryu H, Meikle SR, Willowson KP, Eslick EM, Bailey DL. Performance evaluation of quantitative SPECT/CT using NEMA NU 2 PET methodology. Phys Med Biol. 2019;64:

27. Toriihara A, Daisaki H, Yamaguchi A, Isogai J, Yoshida K, Tateishi U. Applying standardized uptake value to SPECT/CT: Its usefulness and limitations. J Nucl Med. 2019;60:1134.

Publisher's Note Springer Nature remains neutral with regard to jurisdictional claims in published maps and institutional affiliations. 